Pacific Journal of Mathematics

ROBIN FUNCTIONS AND ENERGY FUNCTIONALS OF 


\title{
ROBIN FUNCTIONS AND ENERGY FUNCTIONALS OF MULTIPLY CONNECTED DOMAINS
}

\author{
P. L. Duren ANd M. M. Schiffer
}

The Robin function of a planar domain is a generalization of Green's function. It can be used to represent the solutions of mixed boundary-value problems for harmonic functions. Here it is combined with a variational method to solve certain extremal problems for the energy functional of a multiply connected domain. Some deeper properties of the Robin function are then explored. An allied system of conformal invariants called the Robin matrix is introduced and is compared with the classical Riemann matrix of a finitely connected domain.

In potential theory one considers three important boundary-value problems: to determine a harmonic function $u$ in a given domain from its values on the boundary, from the values of its normal derivative on the boundary, and from the values of $\partial u / \partial n+h(s) u$ on the boundary, where $h(s)$ is a given positive function of the arclength. The problems are solved by means of Green's function, Neumann's function, and Green's function of the third kind, which is sometimes called the Robin function.

In the two-dimensional case, Green's function plays an important role in the theory of conformal mapping because it is conformally invariant. There is now a special type of Robin function which is likewise conformally invariant and leads to additional invariants and interesting applications. We shall define it as the function $R(z, \zeta)$ harmonic in the domain except for a logarithmic pole at $\zeta$, and vanishing on a specified part of the boundary while its normal derivative vanishes on the rest of the boundary.

We begin the paper by recording some of the basic properties of the Robin function. We then establish its existence by displaying it as a solution to a certain extremal problem involving transfinite diameter. Next we use it to solve an extremal problem which arose in our previous study [5] of the energy functional of a multiply connected domain. We also apply it to find the sharp bounds of a quadratic form associated with the Riemann matrix. Finally, we discuss some further properties of the Robin function; for instance, we show that it 
always majorizes Green's function. We conclude by pointing out the role of the Robin function as a reproducing kernel.

The Robin function can be defined similarly in higher-dimensional regions, where it plays a basic role in the solution of boundary-value problems for more general elliptic partial differential equations [1]. Here we shall discuss only harmonic functions in the plane, but some of the theory extends readily to more general situations.

1. Definition and basic properties. Let $\Omega$ be a finitely connected domain in the extended complex plane $\widehat{\mathbb{C}}$, containing the point at infinity and bounded by smooth Jordan curves $\Gamma_{1}, \Gamma_{2}, \ldots, \Gamma_{m}$, where $m \geq 2$. Choose an integer $n$ with $1 \leq n<m$, and divide the boundary $\partial \Omega$ into the two subsets

$$
A=\Gamma_{1} \cup \cdots \cup \Gamma_{n} ; \quad B=\Gamma_{n+1} \cup \cdots \cup \Gamma_{m} .
$$

Then for each $\zeta \in \Omega$, the Robin function is defined by the properties:

(i) $R(z, \zeta)$ is harmonic in $\Omega$ and continuous in $\bar{\Omega}$ together with its first partial derivatives, except at $z=\zeta$, where $R(z, \zeta)+\log |z-\zeta|$ is harmonic;

(ii) $R(z, \zeta)=0$ for all $z \in A$;

(iii) $\frac{\partial R}{\partial n}(z, \zeta)=0$ for all $z \in B$, where $\partial / \partial n$ denotes the inner normal derivative. For $\zeta=\infty$, the property (i) is modified to require that $R(z, \zeta)-\log |z|$ be harmonic in $\Omega$.

The definition of the Robin function and most of the resulting properties are easily generalized to more general complementary subsets $A$ and $B$ of the boundary. The domain may then be simply connected. We have chosen our special definition mainly to focus on certain periods around the boundary components, analogous to the Riemann matrix for harmonic measures, which are important in our applications.

It may also be remarked that the Robin function can be defined (by conformal invariance) even for domains with irregular boundaries where no normal direction exists. Such a finitely connected domain can be mapped conformally onto a domain bounded by analytic Jordan curves, where the Robin function is uniquely determined. The mapping then induces a well-defined Robin function in the original domain.

It is not obvious a priori that the Robin function exists. Deferring a proof to $\S 2$, we shall first develop some of its basic properties.

We begin with the symmetry relation

$$
R(z, \zeta)=R(\zeta, z), \quad z, \zeta \in \Omega \text {. }
$$


For a proof, fix distinct finite points $\zeta, \eta \in \Omega$ and consider the integral

$$
I(\zeta, \eta)=\frac{1}{2 \pi} \int_{\partial \Omega}\left[R(z, \zeta) \frac{\partial R}{\partial n}(z, \eta)-R(z, \eta) \frac{\partial R}{\partial n}(z, \zeta)\right]|d z| .
$$

By properties (ii) and (iii), it is clear that $I(\zeta, \eta)=0$. On the other hand, deforming the path of integration to two small circles around $\zeta$ and $\eta$ and using the property (i), one finds that

$$
I(\zeta, \eta)=R(\eta, \zeta)-R(\zeta, \eta)
$$

hence $R(\eta, \zeta)=R(\zeta, \eta)$. An obvious modification of the argument handles the case $\zeta=\infty$. Alternatively, one can deduce it from the finite case by the mapping $z \mapsto 1 /(z-\zeta)$, since the Robin function is conformally invariant.

The most important property of the Robin function is the formula

$$
u(\zeta)=\frac{1}{2 \pi} \int_{A} \frac{\partial R}{\partial n}(z, \zeta) u(z)|d z|-\frac{1}{2 \pi} \int_{B} R(z, \zeta) \frac{\partial u}{\partial n}(z)|d z|
$$

which represents a function $u$ harmonic in $\bar{\Omega}$ in terms of its boundary values on $A$ and its normal derivative on $B$. In view of (ii) and (iii), the formula (2) is equivalent to

$$
u(\zeta)=\frac{1}{2 \pi} \int_{\partial \Omega}\left[\frac{\partial R}{\partial n}(z, \zeta) u(z)-R(z, \zeta) \frac{\partial u}{\partial n}(z)\right]|d z|,
$$

which is derived in the same way that the symmetry relation (1) is proved.

Conversely, by standard techniques (cf. Nehari [10], pp. 357-360) it can be shown more generally that the formula (2) represents the harmonic function $u$ with prescribed continuous boundary values on $A$ and with prescribed continuous normal derivative on $B$.

The Robin function also allows the representation of a harmonic function in terms of its values on one part of the boundary and the values of its conjugate on the rest of the boundary. Let $u(z)+i v(z)$ be a single-valued analytic function in $\bar{\Omega}$. Start with the formula (2), introduce the general Cauchy-Riemann equation $\partial u / \partial n=\partial v / \partial s$, and integrate the second term by parts to obtain

$$
u(\zeta)=\frac{1}{2 \pi} \int_{A} \frac{\partial R}{\partial n}(z, \zeta) u(z)|d z|+\frac{1}{2 \pi} \int_{B} \frac{\partial R}{\partial s}(z, \zeta) v(z)|d z|,
$$

where $\partial / \partial s$ indicates the tangential derivative. 
We now define the functions

$$
\sigma_{j}(\zeta)=\frac{1}{2 \pi} \int_{\Gamma_{j}} \frac{\partial R}{\partial n}(z, \zeta)|d z|, \quad 1 \leq j \leq n,
$$

which we call the generalized harmonic measures. It is clear from (2) that $\sigma_{j}$ is the harmonic function uniquely determined by the properties

$$
\sigma_{j}(z) \equiv 1 \text { on } \Gamma_{j} ; \quad \sigma_{j}(z) \equiv 0 \text { on } \Gamma_{k}, \quad 1 \leq k \leq n, k \neq j ;
$$

and

$$
\frac{\partial \sigma_{j}}{\partial n}(z)=0 \text { on } \Gamma_{k}, n<k \leq m
$$

The period of the harmonic conjugate of $\sigma_{j}$ around $\Gamma_{k}$ is

$$
Q_{j k}=\frac{1}{2 \pi} \int_{\Gamma_{k}} \frac{\partial \sigma_{j}}{\partial n}(z)|d z| .
$$

Observe that the symmetry relation $Q_{k j}=Q_{j k}$ follows from (1) and (3). Note also that $Q_{j k}=0$ for $n<k \leq m$. The $n \times n$ matrix $\left(\left(Q_{j k}\right)\right)$ will be called the Robin matrix of $\Omega$. It is analogous to the classical Riemann matrix of periods of the harmonic conjugates, to which it reduces in the degenerate case $n=m$.

It will now be shown that the $(n-1) \times(n-1)$ submatrix $\left(\left(Q_{j k}\right)\right)$ with $1 \leq j, k \leq n-1$ is negative definite. Consider for this purpose the harmonic function

$$
H(z)=\sum_{k=1}^{n} x_{k} \sigma_{k}(z),
$$

where the $x_{k}$ are arbitrary real parameters. Invoking (4), (5), and (6), we find

$$
\begin{aligned}
0 & \leq \int_{\Omega}|\nabla H|^{2}(z) d x d y=-\int_{\partial \Omega} H(z) \frac{\partial H}{\partial n}(z)|d z| \\
& =-\sum_{j=1}^{n} \sum_{k=1}^{n} x_{j} x_{k} \sum_{\nu=1}^{m} \int_{\Gamma_{\nu}} \sigma_{j}(z) \frac{\partial \sigma_{k}}{\partial n}(z)|d z| \\
& =-2 \pi \sum_{j=1}^{n} \sum_{k=1}^{n} x_{j} x_{k} Q_{j k} .
\end{aligned}
$$

Thus the quadratic form

$$
\sum_{j=1}^{n} \sum_{k=1}^{n} Q_{j k} x_{j} x_{k} \leq 0
$$


with equality only if $H(z)$ is constant in $\Omega$. But it follows from (4) that $H(z) \equiv x_{k}$ on $\Gamma_{k}$ for $1 \leq k \leq n$; thus the quadratic form vanishes only if $x_{1}=x_{2}=\cdots=x_{n}$. (In fact, it actually vanishes in this case; it is easily shown that

$$
\left.\sum_{j=1}^{n} \sum_{k=1}^{n} Q_{j k}=0 .\right)
$$

In particular, we infer that

$$
\sum_{j=1}^{n-1} \sum_{k=1}^{n-1} Q_{j k} x_{j} x_{k}<0
$$

unless $x_{1}=x_{2}=\cdots=x_{n-1}=0$.

We summarize our findings in the following theorem.

THEOREM 1. Let $\Omega$ be a multiply connected domain with boundary components $\Gamma_{1}, \ldots, \Gamma_{m}$, and let $R(z, \zeta)$ be the Robin function which vanishes on $\Gamma_{1} \cup \cdots \cup \Gamma_{n}$, where $1 \leq n<m$. Then the associated Robin matrix $\left(\left(Q_{j k}\right)\right)$ is symmetric. Furthermore, the quadratic forms (7) and (8) which it generates are negative semidefinite and negative definite, respectively. In particular, the $(n-1) \times(n-1)$ matrix $\left(\left(Q_{j k}\right)\right)$ of $(8)$ is nonsingular. The $Q_{j k}$ are moduli of $\Omega$ since they are conformally invariant.

2. Existence of the Robin function. We shall now prove the existence of the Robin function of the domain $\Omega$. Our strategy is to pose a suitable extremal problem and to show by a variational method that the solution is the Robin function. Another existence proof can be based on the theory of integral equations (cf. [6], p. 622).

First consider the equivalence class $\mathscr{F}(\Omega)$ of domains $D$ obtained from $\Omega$ by conformal mappings $\varphi$ of the form

$$
\varphi(z)=z+\sum_{n=0}^{\infty} b_{n} z^{-n}
$$

near infinity. These mappings will be called admissible. Let $C_{k}=$ $\varphi\left(\Gamma_{k}\right)$ for $k=1,2, \ldots, m$. For $1 \leq n<m$, let $\widehat{R}$ be the transfinite diameter of the set $A=C_{1} \cup \cdots \cup C_{n}$. (See, for instance, Goluzin [7] or Hille [8] for the definition and principal facts about transfinite diameter, or logarithmic capacity.) We pose the extremal problem of minimizing $\widehat{R}$ in the class $\mathscr{F}(\Omega)$. 
Because the admissible mappings $\varphi$ with $b_{0}=0$ constitute a compact normal family, it is clear that a minimizing domain $D \in \mathscr{F}(\Omega)$ exists. Choose $w_{0} \in C_{k}$ for some $k$ with $n<k \leq m$, and introduce the boundary variation

$$
w^{*}=V_{\rho}(w)=w+\frac{a \rho^{2}}{w-w_{0}}+O\left(\rho^{3}\right),
$$

which for small $\rho$ is analytic and univalent in $\widehat{\mathbb{C}}$ minus a small subcontinuum of $C_{k}$ near $w_{0}$. (See [3], Ch. 10.) Thus $\varphi^{*}=V_{\rho} \circ \varphi$ is admissible, and $D^{*}=V_{\rho}(D)$ belongs to the equivalence class $\mathscr{F}(\Omega)$. Let $C_{k}^{*}=V_{\rho}\left(C_{k}\right)$.

Observe now that on the domain $\widehat{D} \supset D$ bounded only by $C_{1}, \ldots$, $C_{n}$, the variation (10) acts as an interior variation. Thus by a known variational formula [12], the transfinite diameter of the perturbed boundary subset $A^{*}=C_{1}^{*} \cup \cdots \cup C_{n}^{*}$ is

$$
\widehat{R}^{*}=\widehat{R}\left[1-\operatorname{Re}\left\{a \rho^{2} \hat{p}^{\prime}\left(w_{0}\right)^{2}\right\}\right]+O\left(\rho^{3}\right),
$$

where $\hat{p}(w)$ is the analytic completion of Green's function $\hat{g}(w)=$ $\hat{g}(w, \infty)$ of $\widehat{D}$. Since $\widehat{R}^{*} \geq \widehat{R}$ by the minimum property of $D$, it follows that

$$
\operatorname{Re}\left\{a \rho^{2} \hat{p}^{\prime}\left(w_{0}\right)^{2}\right\}+O\left(\rho^{3}\right) \leq 0 .
$$

Appealing now to the fundamental lemma of the method of boundary variation ([11]; see also [3], p. 297), we conclude from (11) that the boundary components $C_{n+1}, \ldots, C_{m}$ lie on trajectories of the quadratic differential

$$
\hat{p}^{\prime}(w)^{2} d w^{2}>0 \text {. }
$$

Parametrizing each of these curves $C_{k}$ by $w=w(s)$, we conclude in particular that

$$
\frac{d}{d s} \hat{p}(w(s))=\hat{p}^{\prime}(w(s)) w^{\prime}(s)
$$

is real. In other words, the tangential derivative

$$
\frac{d}{d s} \operatorname{Im}\{\hat{p}(w)\}=0 \text {. }
$$

By the general form of the Cauchy-Riemann equations, it follows from (12) that

$$
\frac{\partial \hat{g}}{\partial n}(w)=\frac{\partial}{\partial n} \operatorname{Re}\{\hat{p}(w)\}=0 \text { on } C_{k}, \quad n<k \leq m .
$$

On the other hand, since $\hat{g}(w)=\hat{g}(w, \infty)$ is Green's function of $\widehat{D}$, it is clear that $\hat{g}(w)=0$ on $C_{k}$ for $1 \leq k \leq n$. From this and 
(13) we see that $\hat{g}(w, \infty)$ is none other than the Robin function of $D$, defined with respect to the boundary subsets $A$ and $B$, with pole at infinity. Thus by conformal invariance, $R(z, \infty)=\hat{g}(\varphi(z), \infty)$ is the Robin function of $\Omega$. This proves the existence of the Robin function with pole at infinity for each smoothly bounded domain $\Omega$. The Robin function $R(z, \zeta)$ is produced through the linear fractional mapping $z \mapsto 1 /(z-\zeta)$.

In particular, we have obtained the following result.

THEOREM 2. Let $\Omega$ be a domain containing infinity, with boundary components $\Gamma_{1}, \ldots, \Gamma_{m}$. Choose an integer $n<m$ and let $\widehat{\Omega} \supset \Omega$ be the domain with boundary $A=\Gamma_{1} \cup \cdots \cup \Gamma_{n}$. Suppose that $\Omega$ is chosen in a given conformal equivalence class such that the transfinite diameter $\widehat{R}$ of $A$ is a minimum. Then Green's function $\hat{g}(z, \infty)$ of $\widehat{\Omega}$ is Robin's function $R(z, \infty)$ of $\Omega$ with respect to $A$.

3. An extremal problem for the energy functional. Suppose again that $\Omega \subset \widehat{\mathbb{C}}$ is a multiply connected domain containing infinity and bounded by smooth Jordan curves $\Gamma_{1}, \Gamma_{2}, \ldots, \Gamma_{m}$. The harmonic measure $\omega_{k}$ is the bounded harmonic function such that $\omega_{k}(z)$ $\equiv 1$ on $\Gamma_{k}$ and $\omega_{k}(z) \equiv 0$ elsewhere on $\partial \Omega$. Note that

$$
\omega_{k}(\infty)=\frac{1}{2 \pi} \int_{\Gamma_{k}} \frac{\partial g}{\partial n}(z)|d z|
$$

where $g(z)=g(z, \infty)$ is Green's function of $\Omega$ with pole at infinity. The period of the harmonic conjugate of $\omega_{j}$ around $\Gamma_{k}$ is

$$
P_{j k}=\frac{1}{2 \pi} \int_{\Gamma_{k}} \frac{\partial \omega_{j}}{\partial n}(z)|d z|
$$

The matrix $\left(\left(P_{j k}\right)\right)$ is called the Riemann matrix. It is known that $P_{k j}=P_{j k}$, and that the $(m-1) \times(m-1)$ submatrix $\left(\left(P_{j k}\right)\right)$ for $j, k=1,2, \ldots, m-1$ is nonsingular; in fact, it generates a negativedefinite quadratic form. (See Nehari [10], Ch. 1.) Thus its inverse matrix $\left(\left(p_{j k}\right)\right)$ has the same properties.

In our previous studies $[4,5]$ of extremal problems for conformal mappings onto nonoverlapping regions, there arose the functional

$$
\psi=\psi(\Omega)=\log R+\sum_{j=1}^{m-1} \sum_{k=1}^{m-1} p_{j k}\left(\omega_{j}(\infty)-x_{j}\right)\left(\omega_{k}(\infty)-x_{k}\right),
$$

where $R$ is the transfinite diameter of the complement $\widetilde{\Omega}$ and $x_{1}, \ldots$, $x_{m-1}$ are nonzero real numbers. Because of its physical significance 
(see [5]), $\psi$ is called the energy functional. It is conformally invariant under all admissible mappings.

The following extremal problem generalizes the one in $\S 2$ which led to the Robin function. Consider again a domain $\Omega$ containing infinity and bounded by $\Gamma_{1}, \ldots, \Gamma_{m}$. Choose an integer $n$ with $1<n<m$, and let $\widehat{\Omega}$ be the larger domain bounded only by $\Gamma_{1}, \ldots, \Gamma_{n}$. Among all domains $\Omega$ in a given equivalence class, we ask for the minimum of the corresponding energy functional

$$
\psi(\widehat{\Omega})=\log \widehat{R}+\sum_{j=1}^{n-1} \sum_{k=1}^{n-1} \hat{p}_{j k}\left(\hat{\omega}_{j}(\infty)-x_{j}\right)\left(\hat{\omega}_{k}(\infty)-x_{k}\right),
$$

where $\widehat{R}$ is the transfinite diameter of the complement of $\widehat{\Omega}$, the harmonic measures $\hat{\omega}_{j}(z)$ and the Riemann matrix $\left(\left(\widehat{P}_{j k}\right)\right)$ are those of $\widehat{\Omega}$, and $\left(\left(\hat{p}_{j k}\right)\right)$ is the inverse of the canonical $(n-1) \times(n-1)$ submatrix of $\left(\left(\widehat{P}_{j k}\right)\right)$.

The existence of an extremal domain is clear. In view of the translation-invariance of $\psi$, it is sufficient to consider only admissible mappings (9) with $b_{0}=0$, and these constitute a normal family. We again denote a minimizing domain by $\Omega$ and its boundary components by $\Gamma_{1}, \ldots, \Gamma_{m}$. We may assume that $\Omega$ is chosen so that $\Gamma_{1}, \ldots, \Gamma_{n}$ are analytic Jordan curves, because $\psi$ is invariant under all admissible mappings of $\widehat{\Omega}$.

In order to describe the extremal domain $\Omega$, we choose a point $z_{0} \in \partial \Omega$ and construct a boundary variation

$$
z^{*}=V_{\rho}(z)=z+\frac{a \rho^{2}}{z-z_{0}}+O\left(\rho^{3}\right),
$$

which for small $\rho$ is analytic and univalent in the entire plane except for a small subcontinuum of $\partial \Omega$ around $z_{0}$. Let $\Omega^{*}=V_{\rho}(\Omega)$ and $\widehat{\Omega}^{*}=V_{\rho}(\widehat{\Omega})$ be the corresponding perturbations, and let $\psi^{*}=\psi\left(\widehat{\Omega}^{*}\right)$ be the induced perturbation of the energy functional $\psi=\psi(\widehat{\Omega})$.

Observe that $V_{\rho}$ is an admissible mapping, so that $\Omega^{*} \in \mathscr{F}(\Omega)$ and $\psi^{*} \geq \psi$. If $z_{0} \in \partial \widehat{\Omega}$, then $\widehat{\Omega}^{*} \in \mathscr{F}(\widehat{\mathbf{\Omega}})$ and so $\psi^{*}=\psi$, by the conformal invariance of the energy functional. However, if $z_{0} \notin \partial \widehat{\Omega}$, but instead $z_{0} \in \Gamma_{k}$ for some $k>n$, then the variation (17) acts as an interior on $\widehat{\Omega}$. The variational formula for $\psi$, as developed at the end of our previous paper [5], now takes the form

$$
\psi^{*}=\psi-\operatorname{Re}\left\{a \rho^{2} h^{\prime}\left(z_{0}\right)^{2}\right\}+O\left(\rho^{3}\right),
$$


where

$$
h(z)=\hat{p}(z)-\sum_{j=1}^{n-1} \hat{v}_{j}(z)\left(\hat{\omega}_{j}(\infty)-x_{j}\right)
$$

and

$$
\hat{v}_{j}(z)=\sum_{l=1}^{n-1} \hat{p}_{j l} \hat{w}_{l}(z)
$$

Here $\hat{p}(z)$ is the analytic completion of Green's function $\hat{g}(z)=$ $\hat{g}(z, \infty)$ of $\widehat{\Omega}$, and $\hat{w}_{j}(z)$ is the analytic completion of $\hat{\omega}_{j}(z)$.

Since $\psi^{*} \geq \psi$, we may again invoke the fundamental lemma of the method of boundary variation to conclude from (18) that each $\Gamma_{k}(n<k \leq m)$ lies on a trajectory of the quadratic differential

$$
h^{\prime}(z)^{2} d z^{2}>0 \text {. }
$$

Taking the square root in (21) and integrating along $\Gamma_{k}$, we arrive at the conditions

$$
\operatorname{Im}\{h(z)\}=\beta_{k}, \quad z \in \Gamma_{k}, n<k \leq m,
$$

where the $\beta_{k}$ 's are constants.

On the other hand, $\operatorname{Re}\{h(z)\}$ is obviously constant on each $\Gamma_{k}$ for $1 \leq k \leq n$. Indeed, $\operatorname{Re}\{\hat{p}(z)\}=0$ and $\operatorname{Re}\left\{\hat{v}_{j}(z)\right\}=\hat{p}_{j k}$ on $\Gamma_{k}$, so that $u(z)=\operatorname{Re}\{h(z)\}$ has the boundary values

$$
u(z)=\alpha_{k}=-\sum_{j=1}^{n-1} \hat{p}_{j k}\left(\hat{\omega}_{j}(\infty)-x_{j}\right), \quad z \in \Gamma_{k}, 1 \leq k \leq n .
$$

Thus the harmonic function

$$
u(z)=\hat{g}(z)-\sum_{j=1}^{n-1} \sum_{k=1}^{n-1} \hat{p}_{j k}\left(\hat{\omega}_{j}(\infty)-x_{j}\right) \hat{\omega}_{k}(z)
$$

is constant on each of the boundary components $\Gamma_{1}, \ldots, \Gamma_{n}$ and has a vanishing normal derivative on $\Gamma_{n+1}, \ldots, \Gamma_{m}$, by (22) and the generalized Cauchy-Riemann equations. It is clear from (24) that $u(z)=0$ on $\Gamma_{n}$; hence $\alpha_{n}=0$. Now introduce the Robin function $R(z, \zeta)$ of $\Omega$ with respect to the boundary sets $A=\Gamma_{1} \cup \cdots \cup \Gamma_{n}$ and $B=\Gamma_{n+1} \cup \cdots \cup \Gamma_{m}$. Thus $R(z, \zeta)=0$ on $A$ and $\partial R(z, \zeta) / \partial n=0$ on $B$. Consider the harmonic function

$$
\varphi(z)=u(z)-R(z, \infty),
$$


and represent it by the basic formula (2):

$$
\varphi(\zeta)=\frac{1}{2 \pi} \int_{A} \frac{\partial R}{\partial n}(z, \zeta) \varphi(z)|d z|-\frac{1}{2 \pi} \int_{B} R(z, \zeta) \frac{\partial \varphi}{\partial n}(z)|d z| .
$$

But we have already observed that $\frac{\partial u}{\partial n}(z)=0$ on $B$, while $u(z)=\alpha_{k}$ on $\Gamma_{k}$ for $1 \leq k \leq n$, where $\alpha_{n}=0$. Thus $\varphi$ has the same properties, and (25) reduces to

$$
u(z)=R(z, \infty)+\sum_{j=1}^{n-1} \alpha_{j} \sigma_{j}(z)
$$

where $\sigma_{j}$ are the generalized harmonic measures of $\Omega$, defined by (3). Integrating the normal derivative $\partial u / \partial n$ and recalling the definition (6) of the periods $Q_{j k}$, we find by (3) and (26)

$$
\frac{1}{2 \pi} \int_{\Gamma_{k}} \frac{\partial u}{\partial n}(z)|d z|=\sigma_{k}(\infty)+\sum_{j=1}^{n-1} \alpha_{j} Q_{j k}, \quad 1 \leq k \leq n .
$$

Similarly, we deduce from (24), (14), and (15) after a short calculation that

$$
\frac{1}{2 \pi} \int_{\Gamma_{k}} \frac{\partial u}{\partial n}(z)|d z|=x_{k}, \quad 1 \leq k \leq n,
$$

where $x_{n}=1-x_{1}-\cdots-x_{n-1}$. Comparing (27) and (28), we arrive at

$$
\sigma_{k}(\infty)+\sum_{j=1}^{n-1} \alpha_{j} Q_{j k}=x_{k}, \quad 1 \leq k \leq n-1 .
$$

This allows us to determine the constants $\alpha_{j}$. By Theorem 1 , the $(n-1) \times(n-1)$ matrix $\left(\left(Q_{j k}\right)\right)$ is nonsingular. Let $\left(\left(q_{j k}\right)\right)$ denote its inverse matrix. Then it follows from (29) that

$$
\alpha_{k}=\sum_{j=1}^{n-1}\left(x_{j}-\sigma_{j}(\infty)\right) q_{j k}, \quad 1 \leq k \leq n-1 .
$$

On the other hand, if we equate the two formulas (24) and (26) for $u(z)$ and let $z$ tend to infinity, we find after introducing (30) that

$$
\begin{aligned}
\log \widehat{R} & +\sum_{j=1}^{n-1} \sum_{k=1}^{n-1} \hat{p}_{j k}\left(\hat{\omega}_{j}(\infty)-x_{j}\right) \hat{\omega}_{k}(\infty) \\
& =\log R_{A}+\sum_{j=1}^{n-1} \sum_{k=1}^{n-1} q_{j k}\left(\sigma_{j}(\infty)-x_{j}\right) \sigma_{k}(\infty),
\end{aligned}
$$


where

$$
-\log R_{A}=\lim _{z \rightarrow \infty}[R(z, \infty)-\log |z|] .
$$

The conformal invariant $R_{A}$ will be called the Robin capacity of the set $A \subset \partial \Omega$ with respect to which the Robin function is defined. Our derivation of (31) used the fact that the transfinite diameter (or logarithmic capacity) $\widehat{R}$ of $\partial \widehat{\Omega}$ is determined by

$$
-\log \widehat{R}=\lim _{z \rightarrow \infty}[\hat{g}(z, \infty)-\log |z|] .
$$

We can now compute the energy functional $\psi(\widehat{\Omega})$ for the extremal domain $\widehat{\Omega}$. A short calculation based on (16) and (31), together with the two expressions for $\alpha_{k}$ given by (23) and (30), leads finally to the formula $\psi(\widehat{\Omega})=\Phi(\Omega)$, where

$$
\Phi(\Omega)=\log R_{A}+\sum_{j=1}^{n-1} \sum_{k=1}^{n-1} q_{j k}\left(\sigma_{j}(\infty)-x_{j}\right)\left(\sigma_{k}(\infty)-x_{k}\right)
$$

Observe that the functional $\Phi$ is conformally invariant and therefore depends only on the equivalence class $\mathscr{F}(\Omega)$. Since $\Omega$ is an extremal domain, we have proved that $\Phi(\Omega)$ is the minimum value of the energy functional $\psi(\widehat{\Omega})$ among all domains $\Omega$ in the given equivalence class. For this reason, we shall call $\Phi$ the subenergy functional of $\Omega$ with respect to $A$. In summary, we have proved the following theorem.

THEOREM 3. Let $\Omega$ be a domain containing infinity and bounded by disjoint continua $\Gamma_{1}, \ldots, \Gamma_{m}$. Given an integer $n$ with $1<n<m$, and given arbitrary nonzero real parameters $x_{1}, \ldots, x_{n-1}$, let $\Phi(\Omega)$ be the subenergy functional of $\Omega$ with respect to the boundary subset $A=\Gamma_{1} \cup \cdots \cup \Gamma_{n}$. Let $\widehat{\Omega} \supset \Omega$ be the domain with boundary $\partial \widehat{\Omega}=A$, and let $\psi(\widehat{\Omega})$ be its energy functional with respect to the parameters $x_{1}, \ldots, x_{n-1}$. Then $\psi(\widehat{\Omega}) \geq \Phi(\Omega)$, and equality is attained for some domain $\Omega$ in each conformal equivalence class.

4. Maximum of the energy functional. Having found the minimum of the energy functional $\psi(\widehat{\Omega})$, we now ask for the maximum value of $\psi(\widehat{\Omega})$ among all domains $\Omega$ in a given equivalence class. Again let $\Omega$ be an extremal domain, and let $\Gamma_{1}, \ldots, \Gamma_{m}$ be its boundary components. The variational method of $\S 3$ may again be applied, and we find in a similar way that for $n<k \leq m$ each $\Gamma_{k}$ lies on an 
orthogonal trajectory of the quadratic differential (21). Thus

$$
h^{\prime}(z)^{2} d z^{2}<0
$$

where $h(z)$ is defined by (19) and (20). Taking the square root in (33) and integrating along $\Gamma_{k}$, we now find that

$$
u(z)=\operatorname{Re}\{h(z)\}=\alpha_{k}, \quad z \in \Omega_{k}, n<k \leq m,
$$

where the $\alpha_{k}$ 's are constants. But it is again obvious from the defining formula (24) that

$$
u(z)=\alpha_{k}, \quad z \in \Gamma_{k}, 1 \leq k \leq n,
$$

where the constants $\alpha_{k}$ are now given by (23), and $\alpha_{n}=0$. Since (24) shows that the harmonic function $u$ has a logarithmic singularity at infinity, it follows from (34) and (35) that

$$
u(z)=g(z)+\sum_{j=1}^{m} \alpha_{j} \omega_{j}(z)
$$

where $g(z)=g(z, \infty)$ and $\omega_{j}(z)$ are Green's function and the harmonic measures of $\Omega$. From (36) we obtain the expressions

$$
\frac{1}{2 \pi} \int_{\Gamma_{k}} \frac{\partial u}{\partial n}(z)|d z|=\omega_{k}(\infty)+\sum_{j=1}^{m} \alpha_{j} P_{j k}, \quad 1 \leq k \leq m,
$$

in view of (14) and (15). Now (37) and (28) give the equations

$$
\sum_{j=1}^{m} \alpha_{j} P_{j k}=x_{k}-\omega_{k}(\infty), \quad 1 \leq k \leq m,
$$

where $x_{k}=0$ for $n<k \leq m$. Since $\sum_{j=1}^{m} P_{j k}=0$, the equations (38) imply

$$
\sum_{j=1}^{m-1}\left(\alpha_{m}-\alpha_{j}\right) P_{j k}=\omega_{k}(\infty)-x_{k}, \quad 1 \leq k \leq m .
$$

The equations (39) are solved by means of the inverse matrix $\left(\left(p_{k l}\right)\right)$ which yields

(40) $\quad \alpha_{m}-\alpha_{k}=\sum_{j=1}^{m-1} p_{j k}\left(\omega_{j}(\infty)-x_{j}\right), \quad 1 \leq k \leq m-1$ 
On the other hand, if we equate the two forms (24) and (36) of $u(z)$ and let $z$ tend to infinity, we find

$$
\begin{aligned}
\log \widehat{R} & +\sum_{j=1}^{n-1} \sum_{k=1}^{n-1} \hat{p}_{j k}\left(\hat{\omega}_{j}(\infty)-x_{j}\right) \hat{\omega}_{k}(\infty) \\
& =\log R+\sum_{k=1}^{m-1}\left(\alpha_{m}-\alpha_{k}\right) \omega_{k}(\infty)-\alpha_{m} .
\end{aligned}
$$

Recalling the definition (16) of the energy functional, we deduce from (41), (40), and (23) that

$$
\begin{aligned}
\psi(\widehat{\Omega})= & \log R+\sum_{j=1}^{m-1} \sum_{k=1}^{m-1} p_{j k}\left(\omega_{j}(\infty)-x_{j}\right) \omega_{k}(\infty)-\alpha_{m} \\
& -\sum_{j=1}^{n-1} \sum_{k=1}^{n-1} \hat{p}_{j k}\left(\hat{\omega}_{j}(\infty)-x_{j}\right) x_{k} \\
= & \log R+\sum_{j=1}^{m-1} \sum_{k=1}^{m-1} p_{j k}\left(\omega_{j}(\infty)-x_{j}\right) \omega_{k}(\infty) \\
& +\sum_{k=1}^{n-1} x_{k} \alpha_{k}-\alpha_{m} .
\end{aligned}
$$

However, because $\alpha_{n}=0, \sum_{k=1}^{n} x_{k}=1$, and $x_{k}=0$ for $n<k \leq m$, it follows from (40) that

$$
\begin{aligned}
& \sum_{k=1}^{n-1} x_{k} \alpha_{k}-\alpha_{m}=\sum_{k=1}^{m-1} x_{k}\left(\alpha_{k}-\alpha_{m}\right) \\
& =-\sum_{j=1}^{m-1} \sum_{k=1}^{m-1} p_{j k}\left(\omega_{j}(\infty)-x_{j}\right) x_{k} .
\end{aligned}
$$

Introducing (43) into (42), we conclude that $\psi(\widehat{\Omega})=\psi(\Omega)$, where $x_{k}=0$ for $n<k \leq m$ in the definition of $\psi(\Omega)$. Since $\Omega$ was an extremal domain, we have therefore established the sharp inequality $\psi(\widehat{\Omega}) \leq \psi(\Omega)$ for all domains $\Omega$ in the given equivalence class. This result may be summarized as follows.

THEOREM 4. Let $\Omega$ be a domain containing infinity, with boundary components $\Gamma_{1}, \ldots, \Gamma_{m}$. For fixed $n$ with $1 \leq n<m$, let $\widehat{\Omega} \supset \Omega$ be the domain with boundary $\partial \widehat{\Omega}=\Gamma_{1} \cup \cdots \cup \Gamma_{n}$. Given a set of nonzero 
real parameters $x_{1}, \ldots, x_{n}$ with sum 1 , let $\psi(\widehat{\Omega})$ be the corresponding energy functional; and let $\psi(\Omega)$ be the energy functional of $\Omega$ with parameters $x_{1}, \ldots, x_{m-1}$, where $x_{k}=0$ for $n<k<m$. Then $\psi(\widehat{\mathbf{\Omega}}) \leq \psi(\Omega)$, and equality is attained for some domain $\Omega$ in each equivalence class.

As a corollary to the proof of Theorems 3 and 4, we can now make a surprising observation concerning the relation between the domains $\Omega$ and $\widehat{\Omega}$ in the extremal situations. Given an arbitrary domain $\widehat{\Omega}$ containing infinity and bounded by smooth curves $\Gamma_{1}, \ldots, \Gamma_{n}$, consider the harmonic function $u(z)$ defined by (24) with arbitrary real parameters $x_{1}, \ldots, x_{n-1}$. Draw $(m-n)$ disjoint arcs $\Gamma_{k}$ along level sets $u(z)=\alpha_{k}, k=n+1, \ldots, m$. Suppose that none of the new slits $\Gamma_{k}$ meets any of the given curves $\Gamma_{1}, \ldots, \Gamma_{n}$, so that the continua $\Gamma_{1}, \ldots, \Gamma_{m}$ bound an $m$-tuply connected domain $\Omega$ containing infinity. Then $\widehat{\Omega}$ maximizes the energy functional $\psi$ in the equivalence class $\mathscr{F}(\Omega)$ with the prescribed $x_{k}$. If instead we draw the arcs $\Gamma_{n+1}, \ldots, \Gamma_{m}$ along level sets $\tilde{u}(z)=\beta_{k}$ of the harmonic conjugate, so that $\partial u / \partial n=0$ there, then $\widehat{\Omega}$ minimizes $\psi$ in $\mathscr{F}(\Omega)$.

Indeed, if $\Gamma_{n+1}, \ldots, \Gamma_{m}$ lie on level sets of $u$, the calculation given in the proof of Theorem 4 shows that $\psi(\widehat{\Omega})=\psi(\Omega)$, where $x_{1}+\cdots+$ $x_{n}=1$ and $x_{k}=0$ for $n<k<m$; and by Theorem 4 this is the maximum value of $\psi$ in $\mathscr{F}(\Omega)$. Similarly, if $\partial u / \partial n=0$ on $\Gamma_{n+1}, \ldots, \Gamma_{m}$, it follows from the proof of Theorem 3 that $\psi(\widehat{\Omega})=$ $\Phi(\Omega)$, the minimum value in $\mathscr{F}(\Omega)$.

5. Sharp bounds for a quadratic form. Theorems 3 and 4 provide the sharp inequalities $\Phi(\Omega) \leq \psi(\widehat{\Omega}) \leq \psi(\Omega)$. There is an interesting consequence. Let $x_{k}=t \xi_{k}, k=1, \ldots, n-1$; then $x_{n}=1-t \sigma$, where $\sigma=\xi_{1}+\cdots+\xi_{n-1}$. Making these substitutions in the above inequalities, dividing by $t^{2}$, and letting $t$ tend to infinity, one finds after a simple calculation that

$$
\sum_{j=1}^{n-1} \sum_{k=1}^{n-1} q_{j k} \xi_{j} \xi_{k} \leq \sum_{j=1}^{n-1} \sum_{k=1}^{n-1} \hat{p}_{j k} \xi_{j} \xi_{k}
$$

and

$$
\sum_{j=1}^{n-1} \sum_{k=1}^{n-1} \hat{p}_{j k} \xi_{j} \xi_{k} \leq \sum_{j=1}^{n-1} \sum_{k=1}^{n-1} p_{j k} \xi_{j} \xi_{k}-2 \sigma \sum_{j=1}^{n-1} p_{j n} \xi_{j}+\sigma^{2} p_{n n}
$$

for arbitrary real parameters $\xi_{1}, \ldots, \xi_{n-1}$. 
The inequalities (44) and (45) suggest the reverse inequalities for the original matrices $\left(\left(Q_{j k}\right)\right),\left(\left(\widehat{P}_{j k}\right)\right)$, and $\left(\left(P_{j k}\right)\right)$. Indeed, we shall prove the following theorem.

THEOREM 5. Let $\Omega \subset \widehat{\mathbb{C}}$ be a multiply connected domain containing infinity, with smooth boundary curves $\Gamma_{1}, \ldots, \Gamma_{m}$. For fixed $n$ with $1 \leq n<m$, let $\widehat{\Omega}$ be the larger domain with boundary $A=\Gamma_{1} \cup \cdots \cup \Gamma_{n}$. Let $\left(\left(P_{j k}\right)\right)$ and $\left(\left(\widehat{P}_{j k}\right)\right)$ be the Riemann matrices of $\Omega$ and $\widehat{\Omega}$, and let $\left(\left(Q_{j k}\right)\right)$ be the Robin matrix of $\Omega$ with respect to $A$. Then for each choice of real parameters $x_{1}, \ldots, x_{n}$, the inequalities

$$
\sum_{j=1}^{n} \sum_{k=1}^{n} P_{j k} x_{j} x_{k} \leq \sum_{j=1}^{n} \sum_{k=1}^{n} \widehat{P}_{j k} x_{j} x_{k} \leq \sum_{j=1}^{n} \sum_{k=1}^{n} Q_{j k} x_{j} x_{k}
$$

hold. Among all domains $\Omega$ in each given conformal equivalence class, the right-hand inequality in (46) is sharp.

REMARKS. For $x_{n}=0$, the right-hand inequality in (46) can be deduced algebraically from the inequality (44) for the inverse matrices, using the fact (see [2], p. 37) that two symmetric nonsingular matrices can be simultaneously diagonalized. Conversely, Theorem 4 implies both (44) and (45) with the last two terms excised. We shall give a variational proof of (46) which actually identifies the sharp lower bound and thus leads to a stronger result than we stated in the theorem.

Proof of Theorem. Note that the matrices $\left(\left(P_{j k}\right)\right)$ and $\left(\left(Q_{j k}\right)\right)$ are conformally invariant under all admissible mappings of $\Omega$, but $\left(\left(\widehat{P}_{j k}\right)\right)$ is not. Holding $x_{1}, \ldots, x_{n}$ fixed, we ask first for the maximum value of the functional

$$
\phi=\phi(\Omega)=\sum_{j=1}^{n} \sum_{k=1}^{n} \widehat{P}_{j k} x_{j} x_{k}
$$

among all domains $\Omega$ in the given equivalence class. As in the proofs of Theorems 3 and 4, the existence of an extremal domain is clear from the translation-invariance of $\phi$. Again denote the extremal domain by $\Omega$ and its boundary components by $\Gamma_{1}, \ldots, \Gamma_{m}$.

Choose $z_{0} \in \Gamma_{k}$ for some $k>n$ and consider again the boundary variation $z^{*}=V_{\rho}(z)$ given by (17). This is an admissible mapping of $\Omega$, sending it to $\Omega^{*}=V_{\rho}(\Omega)$, and it acts as an interior variation of $\widehat{\Omega}$. The variational formula for the period $\widehat{P}_{j k}$ is known [12] to be

$$
\widehat{P}_{j k}^{*}=\widehat{P}_{j k}+\operatorname{Re}\left\{a \rho^{2} \hat{w}_{j}^{\prime}\left(z_{0}\right) \hat{w}_{k}^{\prime}\left(z_{0}\right)\right\}+O\left(\rho^{3}\right),
$$


where $\hat{w}_{j}(z)$ is the analytic completion of the harmonic measure $\hat{\omega}_{j}(z)$ of $\Gamma_{j}$ with respect to $\widehat{\Omega}$. Applying the formula (47), we see that the maximum value $\phi=\phi(\Omega)$ of the functional is perturbed to $\phi^{*}=\phi\left(\Omega^{*}\right)$ given by

$$
\phi^{*}=\phi+\operatorname{Re}\left\{a \rho^{2} F^{\prime}\left(z_{0}\right)^{2}\right\}+O\left(\rho^{3}\right),
$$

where

$$
F(z)=\sum_{j=1}^{n} x_{j} \hat{w}_{j}(z)
$$

Since $\phi^{*} \leq \phi$, we may again invoke the fundamental lemma of the method of boundary variation to conclude from (48) that each $\Gamma_{k}$ with $n<k \leq m$ lies on a trajectory of the quadratic differential

$$
F^{\prime}(z)^{2} d z^{2}>0 \text {. }
$$

Taking the square root of (50) and integrating, we therefore find that $F(z)$ has constant imaginary part on each curve $\Gamma_{k}$ :

$$
\operatorname{Im}\{F(z)\}=\beta_{k}, \quad z \in \Gamma_{k}, \quad n<k \leq m .
$$

On the other hand, it is clear from (49) that $F(z)$ has constant real part on each $\Gamma_{k}$ with $1 \leq k \leq n$ :

$$
U(z)=\operatorname{Re}\{F(z)\}=x_{k}, \quad z \in \Gamma_{k}, \quad 1 \leq k \leq n .
$$

By the general form of the Cauchy-Riemann equations, we may infer from (51) that $\partial U / \partial n(z)=0$ on each curve $\Gamma_{k}, n<k \leq m$. Thus we see that

$$
U(z)=\sum_{j=1}^{n} x_{j} \hat{\omega}_{j}(z)=\sum_{j=1}^{n} x_{j} \sigma_{j}(z),
$$

where $\sigma_{j}$ are the generalized harmonic measures of $\Omega$ with respect to $A$, defined by (3). Now integrate the normal derivative $\partial U / \partial n$ around $\Gamma_{k}$ to conclude from (53) that

$$
\sum_{j=1}^{n} x_{j} \widehat{P}_{j k}=\sum_{j=1}^{n} x_{j} Q_{j k}, \quad 1 \leq k \leq n .
$$

Multiplying (54) by $x_{k}$ and summing over $k$, we arrive finally at the formula

$$
\phi=\sum_{j=1}^{n} \sum_{k=1}^{n} Q_{j k} x_{j} x_{k}
$$


for the extremal domain. Since this is the maximum value, we have established the upper bound (46) and we have proved that equality is attained for some domain $\Omega$ in each prescribed equivalence class.

Next let $\Omega$ be a domain which minimizes the functional $\phi$ in a given equivalence class. The variational method then leads to the information that

$$
\operatorname{Re}\{F(z)\}=\alpha_{k}, \quad z \in \Gamma_{k}, n<k \leq m .
$$

The conditions (53) and (55) now lead to the equation

$$
U(z)=\sum_{j=1}^{n} x_{j} \hat{\omega}_{j}(z)=\sum_{j=1}^{n} x_{j} \omega_{j}(z)+\sum_{j=n+1}^{m} \alpha_{j} \omega_{j}(z),
$$

where $\omega_{j}$ is the harmonic measure of $\Gamma_{j}$ with respect to $\Omega$. From (56) we conclude as before by comparing periods of $\widetilde{U}$ around $\Gamma_{k}$ that

$$
\sum_{j=1}^{n} x_{j} \widehat{P}_{j k}=\sum_{j=1}^{n} x_{j} P_{j k}+\sum_{j=n+1}^{m} \alpha_{j} P_{j k}, \quad 1 \leq k \leq m,
$$

where $\widehat{P}_{j k}=0$ for $n<k \leq m$.

The linear equations (57) may be solved for $\alpha_{j}$ in terms of the $(m-n) \times(m-n)$ matrix $\left(\left(\Pi_{j k}\right)\right)$ which is the inverse of $\left(\left(P_{j k}\right)\right)$ for $n+1 \leq j, k \leq m$. The solution is

$$
\alpha_{j}=-\sum_{l=1}^{n} \sum_{\nu=n+1}^{m} x_{l} P_{l \nu} \Pi_{\nu j}, \quad n<j \leq m .
$$

Now insert (58) into (57), multiply by $x_{k}$, and sum over $k$ to arrive at the formula

$$
\phi=\sum_{j=1}^{n} \sum_{k=1}^{n} P_{j k} x_{j} x_{k}-\sum_{j=n+1}^{m} \sum_{k=n+1}^{m} \Pi_{j k} \xi_{j} \xi_{k},
$$

where

$$
\xi_{j}=\sum_{l=1}^{n} P_{j l} x_{l}, \quad n<j \leq m .
$$

Since (59) is the value of $\phi$ for the extremal domain, it is the sharp lower bound for $\phi(\Omega)$ as $\Omega$ ranges over the given equivalence class. But $\left(\left(\Pi_{j k}\right)\right)$ generates a negative-definite quadratic form, so (59) is actually larger than the lower bound asserted in the theorem unless all $\xi_{j}=0$. This can happen nontrivially only if $m<2 n$, so that the 
number of variables $x_{l}$ exceeds the number of equations in (60), and the $x_{l}$ are chosen to make all of the sums in (60) vanish.

6. Monotonicity properties of the Robin function. A closer study of the Robin function reveals some remarkable properties. We begin by showing that it always majorizes Green's function.

THEOREM 6. Let $\Omega \subset \widehat{\mathbb{C}}$ be a multiply connected domain bounded by smooth curves $\Gamma_{1}, \ldots, \Gamma_{m}$. Let $R(z, \zeta)$ be the Robin function of $\Omega$, defined with respect to the boundary subsets $A=\Gamma_{1} \cup \cdots \cup \Gamma_{n}$ and $B=\Gamma_{n+1} \cup \cdots \cup \Gamma_{m}$; and let $g(z, \zeta)$ be Green's function of $\Omega$. Then $R(z, \zeta)>g(z, \zeta)$ for every point $z \in \Omega, z \neq \zeta$. Furthermore, $R(z, \zeta)>0$ for all $z \in B$; and $\frac{\partial R}{\partial n}(z, \zeta)>0$ for all $z \in A$.

Proof. In view of the conformal invariance of both Green's function and Robin's function, we may assume without loss of generality that the boundary curves are analytic. Observe first that by the maximum principle the harmonic function

$$
H(z, \zeta)=R(z, \zeta)-g(z, \zeta)
$$

takes its minimum value on the boundary of $\Omega$. We claim that it attains its minimum on $A$ and not on $B$. Suppose, on the contrary, that $H(z, \zeta)$ assumes its minimum at some point $z_{0} \in B$. Then the inner normal derivative $\frac{\partial H}{\partial n}\left(z_{0}, \zeta\right) \geq 0$. But $\frac{\partial R}{\partial n}(z, \zeta)=0$ for all $z \in B$, so this implies that $\frac{\partial g}{\partial n}\left(z_{0}, \zeta\right) \leq 0$. However, since $\Omega$ has a $C^{2}$ boundary, it is a well-known consequence of Hopf's lemma [9] that $\frac{\partial g}{\partial n}(z, \zeta)>0$ for all $z \in \partial \Omega$. This contradiction shows that $H(z, \zeta)$ attains its minimum on $A$, and not on $B$. But by definition, $R(z, \zeta) \equiv g(z, \zeta) \equiv 0$ on $A$. Thus $H(z, \zeta) \geq 0$ in $\Omega$, and strict inequality occurs everywhere unless $H(z, \zeta) \equiv 0$. However, it is clear that $H(z, \zeta) \not \equiv 0$, since $\frac{\partial R}{\partial n}(z, \zeta)=0$ on $B$, while $\frac{\partial g}{\partial n}(z, \zeta)>0$ everywhere on $\partial \Omega$, again by Hopf's lemma. This proves that $R(z, \zeta)>g(z, \zeta)$ everywhere in $\Omega$ and on $B$. In particular, $R(z, \zeta)>0$ on $B$, while $\frac{\partial R}{\partial n}(z, \zeta) \geq \frac{\partial g}{\partial n}(z, \zeta)>0$ on $A$. This completes the proof.

In fact, the Robin function exhibits a monotonicity property with respect to the boundary subset $A$ on which it vanishes. This principlề is expressed by the following theorem.

THEOREM 7. Let $\Omega \subset \widehat{\mathbb{C}}$ be a multiply connected domain bounded by smooth Jordan curves $\Gamma_{1}, \ldots, \Gamma_{m}$. For $1 \leq n<m$, let $R_{n}(z, \zeta)$ 
be the Robin function of $\Omega$ which vanishes on $A_{n}=\Gamma_{1} \cup \cdots \cup \Gamma_{n}$ and has vanishing normal derivative on $B_{n}=\Gamma_{n+1} \cup \cdots \cup \Gamma_{m}$. Then $R_{n+1}(z, \zeta)<R_{n}(z, \zeta)$ for every point $z \in \Omega, z \neq \zeta$, and for every $z \in B_{n}$. Furthermore, $\partial R_{n+1} / \partial n(z, \zeta)<\partial R_{n} / \partial n(z, \zeta)$ for every $z \in A_{n}$.

Proof. Again we may assume, by conformal invariance, that the boundary curves are analytic. We may also assume that $n \leq m-$ 2 (hence that $m \geq 3$ ), since $R_{m}(z, \zeta)$ is Green's function and so the case $n=m-1$ is covered by Theorem 6 . By definition, $R_{n}(z, \zeta)=R_{n+1}(z, \zeta)=0$ for all $z \in A_{n}$ and $\partial R_{n} / \partial n(z, \zeta)=$ $\partial R_{n+1} / \partial n(z, \zeta)=0$ for all $z \in B_{n+1}$; while $R_{n+1}(z, \zeta)=0$ and $\partial R_{n} / \partial n(z, \zeta)=0$ for all $z \in \Gamma_{n+1}$. Thus for fixed $\zeta$, the harmonic function

$$
H(z)=R_{n}(z, \zeta)-R_{n+1}(z, \zeta)
$$

vanishes on $A_{n}$ and has vanishing normal derivative on $B_{n+1}$; while $H(z)>0$ on $\Gamma_{n+1}$, by Theorem 6 . Note in particular that $H(z)$ is not constant in $\Omega$.

Let us ask for the minimum of $H(z)$ in the closure of $\Omega$. We claim that the minimum cannot be attained on $B_{n+1}$. If it were, then because $\partial H / \partial n=0$ there it would follow from the general form of Hopf's lemma [9] that $H(z)$ is constant in $\Omega$, which is not the case. Thus the minimum is attained on $A_{n}$, which implies that $H(z) \geq 0$ in the closure of $\Omega$. It now follows from the maximum principle for harmonic functions that $H(z)>0$ for all $z \in \Omega$. Furthermore, $H(z)>0$ on $B_{n+1}$, since $H(z)$ does not reach its minimum there. Finally, another application of Hopf's lemma shows that $\partial H / \partial n(z)>$ 0 on $A_{n}$, since $H(z)=0$ there. This completes the proof.

Finally, Theorem 7 may be applied to derive a monotonicity property of the period matrix $\left(\left(Q_{j k}\right)\right)$. In the notation of Theorem 6 , let

$$
\sigma_{j}^{(n)}(\zeta)=\frac{1}{2 \pi} \int_{\Gamma_{j}} \frac{\partial R_{n}}{\partial n}(z, \zeta)|d z|, \quad 1 \leq j \leq n,
$$

be the generalized harmonic measures with respect to $A_{n}$, and let

$$
Q_{j k}^{(n)}=\frac{1}{2 \pi} \int_{\Gamma_{k}} \frac{\partial \sigma_{j}^{(n)}}{\partial n}(z)|d z|, \quad 1 \leq j, k \leq n,
$$


denote the Robin matrix of $\Omega$ with respect to $A_{n}$. Since Green's function $g(z, \zeta)=R_{m}(z, \zeta)$, it is clear that $\left(\left(Q_{j k}^{(m)}\right)\right)$ is simply the Riemann matrix $\left(\left(P_{j k}\right)\right)$ of $\Omega$.

THEOREM 8. Let $\Omega$ be a multiply connected domain with smooth boundary curves $\Gamma_{1}, \ldots, \Gamma_{m}$. In the above notation, let $\left(\left(Q_{j k}^{(n)}\right)\right)$ denote the $n \times n$ Robin matrix of $\Omega$ with respect to $A_{n}$. Then for $1 \leq n<m$ the strict inequalities

$$
Q_{j k}^{(n+1)}<Q_{j k}^{(n)}, \quad 1 \leq j, k \leq n,
$$

hold. In particular, $P_{j k}<Q_{j k}^{(n)}$, where $\left(\left(P_{j k}\right)\right)$ is the Riemann matrix of $\Omega$.

Proof. According to Theorem 7,

$$
\frac{\partial R_{n+1}}{\partial n}(z, \zeta)<\frac{\partial R_{n}}{\partial n}(z, \zeta), \quad z \in A_{n} .
$$

Thus it is clear from (61) that $\sigma_{j}^{(n+1)}(z)<\sigma_{j}^{(n)}(z)$ for all $z \in \Omega$ if $1 \leq j \leq n$. Recall now that $\sigma_{j}^{(n)}$ is the harmonic function for which $\sigma_{j}^{(n)}(z) \equiv 1$ on $\Gamma_{j}, \sigma_{j}^{(n)} \equiv 0$ on $\Gamma_{k}$ for $1 \leq k \leq n$ but $k \neq j$, and $\partial \sigma_{j}^{(n)}(z) / \partial n \equiv 0$ on $\Gamma_{k}$ for $n<k \leq m$. Thus the harmonic function

$$
u(z)=\sigma_{j}^{(n)}(z)-\sigma_{j}^{(n+1)}(z)
$$

is positive in $\Omega$ and vanishes everywhere on $A_{n}$. Thus by Hopf's lemma, $\frac{\partial u}{\partial n}(z)>0$ for all $z \in A_{n}$. This holds in particular on each curve $\Gamma_{k}$ with $1 \leq k \leq n$, so it is clear from the definition (62) that $Q_{j k}^{(n+1)}<Q_{j k}^{(n)}$ for $1 \leq j, k \leq n$. Taking $n=m-1$, we conclude that $P_{j k}<Q_{j k}^{(m-1)}$, and it obviously follows that $P_{j k}<Q_{j k}^{(n)}$ for every $n$ $(1 \leq n<m)$ and for all indices $j$ and $k$ in the range $1 \leq j, k \leq n$.

7. The Robin function as a reproducing kernel. Again let $\Omega$ be a domain bounded by smooth curves $\Gamma_{1}, \ldots, \Gamma_{m}$, and let $R(z, \zeta)$ be the Robin function of $\Omega$ with respect to the boundary subset $A=$ $\Gamma_{1} \cup \cdots \cup \Gamma_{n}$, where $1 \leq n<m$. Let $B=\Gamma_{n+1} \cup \cdots \cup \Gamma_{m}$ be the complementary subset of $\partial \Omega$.

Consider the linear space $N$ of all functions harmonic in $\Omega$ which vanish on $A$ and have a finite Dirichlet integral:

$$
\|u\|^{2}=\iint_{\Omega}|\nabla u(z)|^{2} d x d y<\infty .
$$


Define the kernel

$$
K(z, \zeta)=\frac{1}{2 \pi}[R(z, \zeta)-g(z, \zeta)],
$$

where $g(z, \zeta)$ is Green's function of $\Omega$. Observe that for each fixed $\zeta \in \Omega$, the function $K(z, \zeta)$ belongs to $N$. For each $u \in N$ we have

$$
\begin{aligned}
\iint_{\Omega} & \nabla K(z, \zeta) \cdot \nabla u(z) d x d y \\
& =-\int_{B} \frac{\partial K}{\partial n}(z, \zeta) u(z)|d z| \\
& =\frac{1}{2 \pi} \int_{\partial \Omega} \frac{\partial g}{\partial n}(z, \zeta) u(z)|d z|=u(\zeta) .
\end{aligned}
$$

Thus $K(z, \zeta)$ is a reproducing kernel in $N$. Note that $K(z, \zeta)=$ $K(\zeta, z)$, and that $K(z, \zeta)>0$ by Theorem 6 .

The reproducing property $(63)$ shows that $\|K(z, \zeta)\|^{2}=K(\zeta, \zeta)$. It also follows from (63) and the Schwarz inequality that

$$
u(\zeta)^{2} \leq K(\zeta, \zeta)\|u\|^{2}
$$

and equality occurs for $u(z)=K(z, \zeta) / K(\zeta, \zeta)$. Another consequence of (63) is the uniqueness of the reproducing kernel.

This point of view suggests a new approach to the Robin function. In particular, the direct construction of the kernel function through a complete orthonormal system will give another proof of the existence of the Robin function.

As an example, let us construct the kernel function of the annulus $\Omega=\{z: 1<|z|<b\}$, where $A$ is the inner boundary circle $|z|=1$. A complete orthonormal system in the space $N$ is provided by the functions

$$
\begin{aligned}
u_{0}(z) & =c_{0} \log r \\
u_{2 n}(z) & =c_{2 n}\left(r^{n}-r^{-n}\right) \cos n \theta, \quad n=1,2 \ldots \\
u_{2 n+1}(z) & =c_{2 n+1}\left(r^{n}-r^{-n}\right) \sin n \theta, \quad n=0,1, \ldots,
\end{aligned}
$$

where $z=r e^{i \theta}$ and the positive constants $c_{i}$ are chosen to make $\left\|u_{k}\right\|=1$. A calculation gives

$$
\begin{aligned}
c_{0} & =\{2 \pi \log b\}^{-1 / 2}, \\
c_{2 n} & =c_{2 n+1}=\left\{n \pi\left(b^{2 n}-b^{-2 n}\right)\right\}^{-1 / 2} .
\end{aligned}
$$


Thus the kernel function is

$$
K(z, \zeta)=\frac{\log r \log \rho}{2 \pi \log b}+\sum_{n=1}^{\infty} \frac{\left(r^{n}-r^{-n}\right)\left(\rho^{n}-\rho^{-n}\right)}{n \pi\left(b^{2 n}-b^{-2 n}\right)} \cos n(\theta-\varphi)
$$

where $z=r e^{i \theta}$ and $\zeta=\rho e^{i \varphi}$.

It is well known that Green's function of an annulus can be given explicitly in terms of elliptic functions. Having Green's function and the kernel function, one then has Robin's function of the annulus.

Alternatively, Robin's function of the annulus can be calculated directly by conformal mapping. In terms of Jacobi's sine amplitude function $\operatorname{sn} z$, which maps a rectangle onto the upper half-plane, one can construct a function $w=F(z)$ which maps the annulus $\Omega$ conformally onto a domain $D \subset \widehat{\mathbb{C}}$ with boundary components $C_{1}$ and $C_{2}$, where $C_{1}$ is again the unit circle and $C_{2}$ is a radial segment outside $C_{1}$. The mapping can be constructed so that $C_{1}=F\left(\Gamma_{1}\right)$ and $F(\zeta)=\infty$ for an arbitrarily specified point $\zeta \in \Omega$. It is clear by inspection that the Robin function of $D$ with pole at infinity is simply $\log |w|$. Thus the Robin function of $\Omega$ is $R(z, \zeta)=\log |F(z)|$.

\section{REFERENCES}

[1] S. Bergman and M. Schiffer, Kernel Functions and Elliptic Differential Equations in Mathematical Physics, Academic Press, New York, 1953.

[2] R. Courant and D. Hilbert, Methods of Mathematical Physics, Vol. I, Interscience, New York, 1953.

[3] P. L. Duren, Univalent Functions, Springer-Verlag, Heidelberg and New York, 1983.

[4] P. L. Duren and M. M. Schiffer, Conformal mappings onto nonoverlapping regions, in Complex Analysis (edited by J. Hersch and A. Huber; Birkhäuser Verlag, Basel, 1988), pp. 27-39.

[5] __, Goluzin inequalities and minimum energy for mappings onto nonoverlapping regions, Ann. Acad. Sci. Fenn. Ser. A. I, 15 (1990), 133-150.

[6] Ph. Frank and $\mathrm{R}$. von Mises, Die Differential- und Integralgleichungen der Mechanik und Physik, F. Viewig \& Sohn, Braunschweig, 1930.

[7] G. M. Goluzin, Geometric Theory of Functions of a Complex Variable, Moscow, 1952; German transl., Deutscher Verlag, Berlin, 1957; 2nd ed., Moscow, 1966; English transl., Amer. Math. Soc., 1969.

[8] E. Hille, Analytic Function Theory, Vol. II, Ginn and Co., Boston, 1962; Second " ed.: Chelsea Publ. Co., New York, 1987.

[9] E. Hopf, A remark on linear elliptic differential equations of second order, Proc. Amer. Math. Soc., 3 (1952), 791-793.

[10] Z. Nehari, Conformal Mapping, McGraw-Hill, New York, 1952; Dover reprint, 1975. 
[11] M. Schiffer, $A$ method of variation within the family of simple functions, Proc. London Math. Soc., 44 (1938), 432-449.

[12] _ Hadamard's formula and variation of domain-functions, Amer. J. Math., 68 (1946), 417-448.

Received August 28, 1989. The research of the first-named author was supported in part by the National Science Foundation under Grant DMS-8701751. The paper was written while he was a Visiting Scholar at Stanford University.

UNIVERSITY OF MiCHIGAN

ANN ARBOR, MI 48109

AND

STANFORD UNIVERSITY

STANFORD, CA 94305 



\section{PACIFIC JOURNAL OF MATHEMATICS EDITORS}

\author{
V. S. VARADARAJAN \\ (Managing Editor) \\ University of California \\ Los Angeles, CA 90024-1555-05 \\ Herbert Clemens \\ University of Utah \\ Salt Lake City, UT 84112 \\ THOMAS ENRIGHT \\ University of California, San Diego \\ La Jolla, CA 92093
}

R. FINN

Stanford University

Stanford, CA 94305

HeRmann FlaschKa

University of Arizona

Tucson, AZ 85721

VAUghan F. R. Jones

University of California

Berkeley, CA 94720

SteVEN KeRCKHOFF

Stanford University

Stanford, CA 94305

\section{C. MOORE}

University of California

Berkeley, CA 94720

MARTIN SCHARLEMANN

University of California

Santa Barbara, CA 93106

HAROLD STARK

University of California, San Diego

La Jolla, CA 92093

\section{ASSOCIATE EDITORS \\ R. ARENS \\ E. F. BECKENBACH \\ B. H. NeUmanN \\ F. WoLF \\ (1904-1989) \\ K. Yoshida (1906-1982)}

\section{SUPPORTING INSTITUTIONS}

UNIVERSITY OF ARIZONA

UNIVERSITY OF BRITISH COLUMBIA

CALIFORNIA INSTITUTE OF TECHNOLOGY

UNIVERSITY OF CALIFORNIA

MONTANA STATE UNIVERSITY

UNIVERSITY OF NEVADA, RENO

NEW MEXICO STATE UNIVERSITY

OREGON STATE UNIVERSITY
UNIVERSITY OF OREGON

UNIVERSITY OF SOUTHERN CALIFORNIA

STANFORD UNIVERSITY

UNIVERSITY OF HAWAII

UNIVERSITY OF TOKYO

UNIVERSITY OF UTAH

WASHINGTON STATE UNIVERSITY

UNIVERSITY OF WASHINGTON

The Supporting Institutions listed above contribute to the cost of publication of this Journal, but they are not owners or publishers and have no responsibility for its content or policies.

Mathematical papers intended for publication in the Pacific Journal of Mathematics should be in typed form or offset-reproduced (not dittoed), double spaced with large margins. Please do not use built up fractions in the text of the manuscript. However, you may use them in the displayed equations. Underline Greek letters in red, German in green, and script in blue. The first paragraph must be capable of being used separately as a synopsis of the entire paper. In particular it should contain no bibliographic references. Please propose a heading for the odd numbered pages of less than 35 characters. Manuscripts, in triplicate, may be sent to any one of the editors. Please classify according to the 1991 Mathematics Subject Classification scheme which can be found in the December index volumes of Mathematical Reviews. Supply name and address of author to whom proofs should be sent. All other communications should be addressed to the managing editor, or Elaine Barth, University of California, Los Angeles, California 90024-1555-05.

There are page-charges associated with articles appearing in the Pacific Journal of Mathematics. These charges are expected to be paid by the author's University, Government Agency or Company. If the author or authors do not have access to such Institutional support these charges are waived. Single authors will receive 50 free reprints; joint authors will receive a total of 100 free reprints. Additional copies may be obtained at cost in multiples of 50 .

The Pacific Journal of Mathematics (ISSN 0030-8730) is published monthly except for July and August. Regular subscription rate: $\$ 190.00$ a year (10 issues). Special rate: $\$ 95.00$ a year to individual members of supporting institutions.

Subscriptions, orders for numbers issued in the last three calendar years, and changes of address should be sent to Pacific Journal of Mathematics, P.O. Box 969, Carmel Valley, CA 93924, U.S.A. Old back numbers obtainable from Kraus Periodicals Co., Route 100, Millwood, NY 10546.

The Pacific Journal of Mathematics at P.O. Box 969, Carmel Valley, CA 93924 (ISSN 0030-8730) is published monthly except for July and August. Second-class postage paid at Carmel Valley, California 93924, and additional mailing offices. Postmaster: send address changes to Pacific Journal of Mathématics, P.O. Box 969, Carmel Valley, CA 93924.

PUBLISHED BY PACIFIC JOURNAL OF MATHEMATICS, A NON-PROFIT CORPORATION Copyright (C) 1991 by Pacific Journal of Mathematics 


\section{Pacific Journal of Mathematics}

Vol. 148, No. $2 \quad$ April, 1991

Yuri A. Abramovich, Operators preserving disjointness on rearrangement

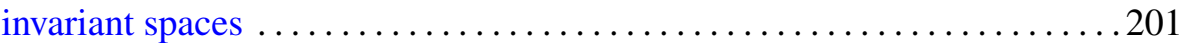

Andrew French Acker and Kirk Lancaster, Existence and geometry of a free boundary problem for the heat equation .................. 207

So-Chin Chen, Real analytic regularity of the Szegő projection on circular

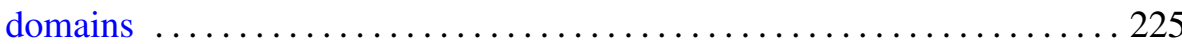

Chen-Lian Chuang, An independence property of central polynomials . . . 237

Peter Larkin Duren and M. Schiffer, Robin functions and energy

functionals of multiply connected domains $\ldots \ldots \ldots \ldots \ldots \ldots \ldots \ldots . \ldots 251$

Johan Henricus Bernardus Kemperman, Sets of uniqueness and systems

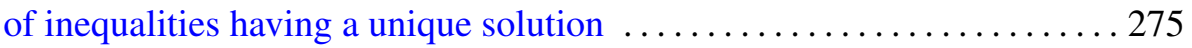

Ka-Lam Kueh, Fourier coefficients of nonholomorphic modular forms and sums of Kloosterman sums . .......................... 303

Gerard J. Murphy, Ordered groups and crossed products of $C^{*}$-algebras . . 319

You-Qiang Wang, The $p$-parts of Brauer character degrees in $p$-solvable

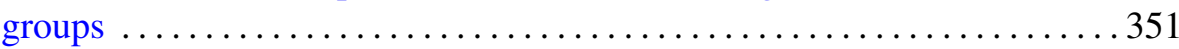

Hidenobu Yoshida, Harmonic majorization of a subharmonic function on a cone or on a cylinder ........................................ 369 